Review Article

\title{
Atypical manifestations of ruptured abdominal aortic aneurysms
}

\author{
A. Banerjee \\ Accident and Emergency Department, East Birmingham Hospital, Bordesley Green East, Birmingham \\ B9 5ST, UK
}

\section{Introduction}

The rupture of an abdominal aortic aneurysm is a catastrophic event with a uniformly fatal outcome if untreated. The triad of abdominal and/or back pain, a pulsatile abdominal mass, and hypotension, is said to be diagnostic. However, this triad may not be present in its entirety, or when present may not be recognized, as one of the components predominates. Clinical diagnosis is thus 'not infrequently missed in the emergency rooms of even the most prestigious medical centers."

To add to this, difficulties in diagnosis may arise owing either to an impalpable aneurysm or to atypical presentations. ${ }^{2,3}$ The clinical diagnosis is often difficult and not infrequently missed. Consequently, appropriate treatment in the form of expeditious laparotomy, rapid control of the proximal aorta and graft replacement of the aneurysmal aorta is not achieved leading to possibly avoidable mortality.

The purpose of this report is to discuss less common presentations of ruptured aneurysms of the abdominal aorta, concentrating on the clinical aspects.

\section{Extent of the problem}

The incidence of abdominal aortic aneurysms appears to be rising, independent of any increased detection from improved diagnostic techniques. ${ }^{4}$ In the absence of any effective community screening programme and also because symptomatic aneurysms are treated surgically at an early stage, ruptured previously undiagnosed aneurysms continue to arrive in hospital. Methods to detect aneurysms based on community screening by clinical examination are, in any case, likely to be inaccurate. ${ }^{5}$ This implies that rupture is often the first clue to the presence of an aneurysm.

Correspondence: A. Banerjee, F.R.C.S.

Accepted: 18 June 1992
The majority of published series of ruptured abdominal aortic aneurysms deal with the outcome of those submitted to surgery. Untreated cases, misdiagnoses and delayed diagnoses are generally not discussed. Hence the precise extent of these problems is unclear.

However, data from various sources suggest that the diagnosis of ruptured abdominal aortic aneurysms is difficult and often missed. In a study of 9.894 autopsies in two Glasgow hospitals, ${ }^{6} 41$ patients were noted to have died in hospital with unoperated ruptured abdominal aortic aneurysms. The diagnostic triad was present in nine. The correct diagnosis had been made in 24 . In a series ofo 100 ruptured aneurysms admitted to hospital, ${ }^{7}$ in? 58 cases surgery was delayed. In 42 instances this was due to an incorrect diagnosis. Emergency operation was performed for an incorrect diagnosis in five cases.

The implications of this are that junior staff may not be prepared to deal with the ruptured aneurysm when encountered and cross-matched blood may not be available.

Of 44 admissions to a hospital with ruptured aneurysms of the abdominal aorta ${ }^{8}$ in 15 patients the diagnosis was missed altogether. In another study of 47 patients with ruptured abdominal aortic aneurysm who died in hospital over a 4 year period, the diagnostic triad was noted in only 19 patients. ${ }^{9}$ Twenty-four patients underwent surgery, only 16 having had the correct preoperative diagnosis.

In a series of 119 patients with ruptured abdominal aortic aneurysms who reached a hospital alive, ${ }^{10} 20$ died in the Accident and Emergency Department (only five being diagnosed correctly), and 24 died on the ward without surgery (nine being undiagnosed).

Of 120 patients with ruptured abdominal aortic aneurysms in one health district, ${ }^{11} 12$ died in hospital from undiagnosed rupture. Of 56 deaths outside hospital, 27 had been seen before death by a doctor but the correct diagnosis was not suspected. 
In the above series, failure to consider the correct diagnosis and to recognize atypical presentations contributed directly to most of the deaths. It is unfortunately not possible to comment on the relative proportions. Inappropriate orthopaedic, urological, neurological and medical referrals resulted in many instances. Commonly recorded misdiagnoses are enumerated in Table I.

\section{Urological presentations}

Urological presentations are not infrequent. Ten per cent of a series of 400 patients with abdominal aortic aneurysms (intact and ruptured) who underwent surgery for the aneurysm presented with features suggesting genitourinary disease. ${ }^{12}$

Unilateral flank pain, either right- or left-sided, with distal radiation to the genitalia, hip and thigh, is often a presenting feature of aneurysmal rupture. This may be misdiagnosed as ureteric colic. ${ }^{13,14}$ The cause of this pain is believed to be irritation of the ureteric pain fibres in the sympathetic plexus by the retroperitoneal haematoma. This appears to be a relatively common diagnostic problem. In one series of 44 cases of aneurysmal rupture, five were noted to have ureteric colic-like presentation. ${ }^{8}$ In another series of 105 patients, 18 presented with unilateral loin pain. ${ }^{15}$

In many cases, the misdiagnosis of ureteric colic leads to inappropriate intravenous urography, with deterioration during, or immediately after, the procedure (Figure 1). One must be wary of the first episode of ureteric colic in the middle aged and elderly, and leaking abdominal aortic aneurysm must be part of the differential diagnosis. In the haemodynamically stable patient, ultrasound assessment is recommended. ${ }^{16}$ This can demonstrate both aneurysmal leakage as well as urinary tract obstruction. Three cases of isolated severe unilateral testicular pain have been reported as the sole presentation of aneurysmal rupture. ${ }^{17}$ The postulated mechanism is haematoma causing pressure on visceral afferent pain fibres from the testes.

Massive haematuria has been described as a

Table I Common misdiagnoses in patients with ruptured abdominal aortic aneurysms

Ureteric colic

Prolapsed lumbar intervertebral disc

Sciatica

Acute myocardial infarction

Perforated peptic ulcer

Acute pancreatitis

Acute cholecystitis

Mesenteric vascular occlusion

Acute diverticulitis presentation of abdominal aortic aneurysmal rupture, in association with fistula formation with either the inferior vena cava or the left renal vein. $^{18-20}$ Haematuria in the presence of a symptomatic aneurysm must be considered as being due to the aneurysm until proven otherwise. Systemic circulatory effects of the fistula must be sought. The haematuria has been attributed to venous congestion of the kidney and bladder, and characteristically clears promptly with resection of the aneurysm.

\section{Neurological presentations}

The precise incidence of neurological complications associated with ruptured abdominal aortic aneurysm is not known. However, they appear to be relatively uncommon. A ruptured abdominal aortic aneurysm can present as an acute unilateral painful neuropathy affecting the femoral and/or obturator nerves. ${ }^{21-25}$ A similar constellation of symptoms and signs has been previously encountered with patients on anticoagulant therapy and in haemophiliacs. The mechanism is that of pressure on these nerves by the extraperitoneal haematoma present in each instance.

The presentation is with severe hip and thigh pain, paraesthesiae and sensory loss of femoral nerve distribution (anteromedial thigh), weakness of hip flexion (iliopsoas) and of knee extension (quadriceps femoris) and loss of the knee jerk. In many reported instances, inappropriate neurological and orthopaedic investigation has been associated with delayed diagnosis. The neuropathy is reversible with early definitive treatment of the aneurysm but, if this is delayed, residual neurological deficit with prominent quadriceps wasting ensues. Intractable sciatica owing to sciatic nerve compression by haematoma has also been described. The presentation has been with severe gluteal pain, restricted straight leg raising and normal neurological examination. ${ }^{26}$ Clearly in the situation with a normal lumbar spine $\mathrm{X}$-ray and aortic calcification, one must be wary of ruptured abdominal aortic aneurysm.

Other reported presentations include lateral popliteal nerve palsy with foot drop (attributed to occlusion of the artery to the sciatic nerve) ${ }^{27}$ and various root compression syndromes. ${ }^{28}$ Acute paraplegia has also been reported as a presentation of aneurysmal rupture ${ }^{27,29}$ although this is more likely to occur as a result of aortic thrombosis or as a postoperative complication. Acute sympathetic paralysis causing a unilateral warm dry foot can occur as a complication of aneurysmal rupture. Haematoma causing damage to the sympathetic ganglia has been implicated as the cause. ${ }^{30}$

The message is that acute painful neuropathies in 
the lower limbs and acute nerve root compression syndromes in the middle aged or elderly can be caused by aortic aneurysmal rupture, which must be considered in the differential diagnosis.

\section{Orthopaedic presentations}

Intractable backache of acute or subacute onset may be the sole presentation of ruptured abdominal aortic aneurysms. This, owing to its frequency cannot be considered to be an atypical presentation. Often, in this instance, diagnostic failure appears to be due to lack of awareness and failure to examine the abdomen.

However, a more chronic form of backache has been described in conjunction with chronic contained ruptures of aortic aneurysms. ${ }^{31-34}$ The special features of this entity are: (a) the presence of abdominal aortic aneurysm; (b) previous pain that may have resolved; (c) a stable patient with normal haematocrit; (d) computed tomography (CT) scan showing retroperitoneal haematoma (soft tissue mass silhouetting psoas muscle, associated with renal displacement); and (e) pathological confirmation of organized haematoma.

A rare presentation with severe chronic backache and systemic symptoms mimicking sepsis in association with vertebral body erosion and disc space narrowing has also been described. ${ }^{35}$ One must beware of chronic intractable backache especially where spinal X-rays do not show a contributory lesion.

\section{Presentations with manifestations of retroperitoneal bleeding}

Ruptured abdominal aortic aneurysms can present with secondary manifestations of extensive retroperitoneal bleeding. They can divert attention from the primary pathological process, although these are seldom isolated presentations.

Rapid onset of a bulging diamond-shaped perianal haematoma has been reported. ${ }^{36-38}$ This is due to extraperitoneal rupture of the aneurysm into the sigmoid mesocolon. The haematoma therefore extends into Waldeyer's fascia and transverses the levator ani muscle sling into the ischiorectal fossa. Localization in the ischiorectal fossa accounts for the characteristic shape. Subsequently tracking across into the subcutaneous perianal space occurs. Retroperitoneal bleeding has been known to produce periumbilical bruising, known as Cullen's sign. ${ }^{39}$ Presentation with Cullen's sign has accordingly been noted with aneurysmal rupture..$^{40}$

Another effect of retroperitoneal bleeding is to cause acute incarceration of a previously reducible inguinal hernia, owing to increased pressure in the inguinal canal. ${ }^{41-44}$ This may lead to inguinal herniorrhaphy as an emergency procedure, when a haemorrhagic hernial sac and/or retroperitoneal tissues may be noted. These, in conjunction with hypotension and anaemia, should suggest the correct diagnosis.

\section{Gastrointestinal presentations}

Abdominal aortic aneurysms may rupture into the gastrointestinal tract, usually into the duodenum $^{45-51}$ or less commonly into the colon. ${ }^{52-53}$ Aortoenteric fistulas are notoriously difficult to diagnose. The triad of abdominal or flank pain, intermittent gastrointestinal bleeding and a pulsatile abdominal mass is diagnostic.

Upper gastrointestinal bleeding is characteristically intermittent, with minor 'herald' or 'sentinel' bleeds preceding an episode of major exsanguinating haemorrhage. Gastrointestinal contrast studies and endoscopy are usually noncontributory. Diagnosis may be confirmed by either flush aortography or CT scanning. Selective arteriography may fail to demonstrate the lesion. Any gastrointestinal bleeding in the presence of a symptomatic aneurysm of the abdominal aorta must be considered as being due to the aneurysm until proven otherwise.

Recurrent lower gastrointestinal bleeding with discharge of fresh blood is the presenting feature of aortocolic fistulas. Abdominal pain and systemic toxicity may be associated. The diagnosis is usually missed and the condition is almost uniformly fatal. Rarely, ruptured aneurysms can present with painful obstructive jaundice ${ }^{54}$ or a cholecystitis-like picture owing to bleeding around the gallbladder..$^{55}$ The latter presentation was said to herald the demise of Albert Einstein.

\section{Cardiovascular complications}

Rupture of the aneurysmal abdominal aorta into either the inferior vena cava or the left renal vein are well described. A total of 159 cases of aortocaval fistula from the world literature were reviewed in a recent article ${ }^{56}$ and this complication was noted to occur with $3-4 \%$ of ruptured abdominal aortic aneurysms. The syndrome of aortocaval fistula is well delineated. ${ }^{57,58}$ It is typical that, in spite of a multitude of physical signs, the diagnosis is missed owing to lack of awareness of the condition.

The features of aortocaval fistula include:

a. Severe abdominal or back pain

b. Systemic venous overload with acute onset high output congestive cardiac failure owing to the large arteriovenous shunt. This causes a 


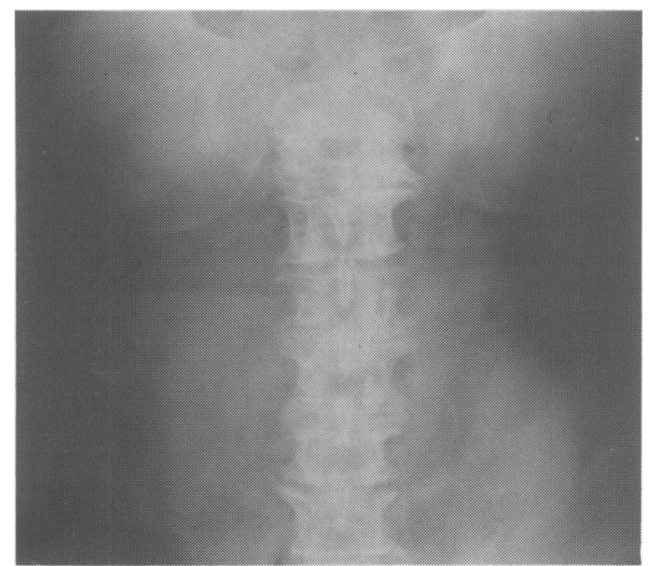

Figure 1 Intravenous urogram showing lateral displacement of left kidney and ureter by ruptured aortic aneurysm. The patient died a few minutes later. (Kindly supplied by Dr D. Hakhamaneshi, M.D., F.R.C.R.)

widened pulse pressure, with reduced diastolic blood pressure, resulting in bounding peripheral pulses, and venous hypertension with a raised jugular venous pressure. The shunt further produces a widely transmitted bruit accompanied by a palpable thrill. The murmur is usually continuous but may be only systolic or absent in the presence of intraluminal thrombosis or venous outflow tract obstruction by the aneurysm. The heart failure is characteristically refractory to the usual anti-failure regimens.

c. Systemic arterial insufficiency causing myocardial ischaemia (angina), ${ }^{59}$ cerebral ischaemic (syncope) and renal insufficiency (oliguria or anuria).

d. Regional venous overload with massive lower limb oedema extending to the lower trunk, haematuria, rectal bleeding and priapism. This constellation can occur in the absence of heart failure by a mechanism of inferior vena caval obstruction. ${ }^{60}$ This causes confusion with retroperitoneal tumours.

e. Paradoxic embolism via fistula into the pulmonary circulation.

f. Branham-Nicoladoni sign of reflex bradycardia on aortic compression, usually noted intraoperatively, but rarely demonstrable preoperatively.

\section{References}

1. Mannick, J.A. \& Whittemore, A.D. Management of ruptured or symptomatic abdominal aortic aneurysms. Surg Clin $N$ Am 1988, 68: 377-384.

2. Szilagyi, D.E., Smith, R.F., Macksood, A.J. \& Whitcomb, J.G. Expanding and ruptured abdominal aortic aneurysms. Problems of diagnosis and treatment. Arch Surg 1961, 83: 395-408.
Table II Suspect ruptured abdominal aortic aneurysm

Unexplained severe abdominal or back pain in middle aged or elderly

Ureteric colic in the elderly

Acute painful femoral neuropathy

Unexplained high output cardiac failure

Unexplained compression of inferior vena cava

Acute incarceration of inguinal hernia

Unexplained gastrointestinal bleeding, especially if recurrent

Unexplained massive haematuria

The clinical diagnosis can usually be confirmed angiographically or by CT scanning.

Aorto-left renal vein fistula is much rarer $^{61-66}$ but can be suspected in the presence of left flank (ureteric colic-like) pain, a pulsatile abdominal mass, haematuria, proteinuria and azotemia. The large majority of reported cases occur in the presence of an anomalous left retro-aortic renal vein which occurs in $2 \%$ of the general population. Left renal vein hypertension may cause rapid onset of a left scrotal varicocele. ${ }^{67}$ Features of high output circulatory state are usually lacking owing to the much smaller shunt. Intravenous urography shows the left kidney to be enlarged and nonfunctioning. Aortography is diagnostic.

\section{Conclusions}

The diagnosis of a ruptured abdominal aortic aneurysm can be difficult. This is partly due to the fact that this condition mimics a variety of other abdominal and extra-abdominal conditions (Table II). Greater awareness of the varied clinical presentations is necessary as well as of the value of urgent ultrasonic assessment in establishing the diagnosis in haemodynamically stable patients.

\section{Acknowledgement}

I wish to thank Mrs A. Danks for kindly typing the manuscript.

3. Gardham, J.R.C. Diagnoses not to be missed. Abdominal aortic aneurysms. Br J Hosp Med 1982, 28: 40-46.

4. Fowkes, F.G.R., Macintyre, C.C.A. \& Ruckley, C.V. Increasing incidence of aortic aneurysms in England and Wales. Br Med J 1989, 298: 33-35. 
5. Allen, P.I.M., Gourevitch, D., McKinley, J., Tudway, D. \& Goldman, M. Population screening for aortic aneurysms (letter). Lancet 1987, ii: 736.

6. McGregor, J.C. Unoperated ruptured abdominal aortic aneurysms: a retrospective clinicopathological study over a 10-year period. Br J Surg 1976, 63: 113-116.

7. Ottinger, L.W. Ruptured arteriosclerotic aneurysms of the abdominal aorta. Reducing mortality. JAMA 1975, 233: $147-150$.

8. Pryer, J.P. Diagnosis of ruptured aneurysm of abdominal aorta. Br Med J 1972, 3: 735-736.

9. Contini, S. \& McMaster, P. Abdominal aortic aneurysm - the forgotten diagnosis? Postgrad Med J 1980, 56: 102-105.

10. Thomas, P.R.S. \& Stewart, R.D. Abdominal aortic aneurysm. Br J Surg 1988, 75: 733-735.

11. Dent, A., Kent, S.J.S. \& Young, T.W. Ruptured abdominal aortic aneurysm. What is the true mortality? (abstract). Br J Surg 1986, 73: 318.

12. Culp, O.S. \& Bernatz, P.E. Urologic aspects of lesions in the abdominal aorta. J Urol 1961, 86: 189-196.

13. Moran, C.G., Edwards, A.T. \& Griffith, G.H. Ruptured abdominal aortic aneurysm presenting with ureteric colic. $\mathrm{Br}$ Med J 1987, 294: 1279.

14. Roussak, J.B. \& Owen, E.R.T.C. Ruptured abdominal aortic aneurysm presenting with ureteric colic (letter). Br Med J 1987, 295: 267.

15. Gaylis, H. \& Kessler, E. Ruptured aortic aneurysms. Surgery 1980, 87: 300-304.

16. Shuman, W.P., Hastrup, W., Jr, Kohler, T.R. et al. Suspected leaking abdominal aortic aneurysm: Use of sonography in the emergency room. Radiology 1988, 168: 117-119.

17. Cawthorn, S.J., Giddings, A.E.B., Taylor, R.S. \& Thomas, M.H. Isolated testicular pain: an unrecognized symptom of the leaking aortic aneurysm. Br J Surg 1991, 78: 886-887.

18. Grahame, J.W. \& Downs, A.R. Ruptured abdominal aortic aneurysm presenting with gross hematuria. J Urol 1971, 106: $628-630$.

19. Brewster, D.C., Ottinger, L.W. \& Darling, R.C. Hematuria as a sign of aorto-caval fistula. Ann Surg 1977, 186: 766-771.

20. van Driel, M., van Gelder, B., Ypma, A. \& van Elk, P. Hematuria as a presenting symptom of an aortic-vena caval fistula from ruptured aortic aneurysm. $J$ Urol 1984, 132: 774-775.

21. Razzuk, M.A., Linton, R.R. \& Darling, R.C. Femoral neuropathy secondary to ruptured abdominal aortic aneurysms with false aneurysms. JAMA 1967, 201: 817-820.

22. Fletcher, H.S. \& Frankel, J. Ruptured abdominal aneurysm presenting with unilateral peripheral neuropathy. Surgery 1976, 79: $120-121$.

23. Merchant, R.F., Cafferata, H.T. \& De Palma, R.G. Ruptured abdominal aortic aneurysm seen initially as acute femoral neuropathy. Arch Surg 1982, 117: 811-813.

24. Owens, M.L. Psoas weakness and femoral neuropathy: Neglected sign of retroperitoneal haemorrhage from ruptured aneurysm. Surgery 1982, 91: 363-364.

25. Marchant, R.F., Cafferata, H.T. \& De Palma, R.G. Pitfalls in the diagnosis of abdominal aortic aneurysm. Am J Surg 1986, 142: $756-758$.

26. Gutman, H., Zelikovski, A., Gadoth, N., Lahav, M. \& Reiss, R. Sciatic pain: A diagnostic pitfall. J Cardiovasc Surg 1987, 28: 204-205.

27. Bolton, P.M. \& Blumgart, L.H. Neurological complications of ruptured aortic aneurysm. Br J Surg 1972, 59: 707-709.

28. Lodder, J., Cheriex, E. \& Oostenbroek, R. Ruptured abdominal aortic aneurysms presenting as radicular compression syndromes. J Neurol 1982, 227: 121-124.

29. Keagy, B.A., Pharr, W.F. \& Bowes, D.E. Unusual presentations of abdominal aortic aneurysms. J Cardiovasc Surg 1981, 22: $41-46$.

30. Eastcott, H.H.G. \& Gardner, A.L. Autosympathectomy in abdominal aortic aneurysm. Ann Surg 1969, 169: 290-291.
31. Jones, C.S., Reilly, M.K., Dalsing, M.C. \& Glover, J.L. Chronic contained rupture of abdominal aortic aneurysms. Arch Surg 1986, 121: 542-546.

32. Chronic contained rupture of abdominal aneurysms (editorial). Lancet 1986, ii: 847-848.

33. Moran, K.T., Persson, A.V. \& Jewell, E.R. Chronic rupture of abdominal aortic aneurysms. Am Surg 1989, 55: 485-487.

34. Clayton, M.J., Walsh, J.W. \& Brewer, W.H. Contained rupture of abdominal aortic aneurysms: Sonographic and CT diagnosis. AJR 1982, 138: 154-156.

35. Choplin, R.H., Karstadet, N. \& Wolfman, N.T. Ruptured abdominal aortic aneurysm simulating pyogenic vertebral spondylitis. $A J R$ 1982, 138: 748-750.

36. Tamvakopoulos, S.K., Corvese, W.P. \& Vargas, L.L. Perianal hematoma-A sign of leakage after rupture of aortic aneurysm. $N$ Engl J Med 1969, 280: 548-549.

37. Tamvakopoulos, S.K., Corvese, W.P. \& Vargas, L.L. Perianal ecchymosis as a clinical sign. Arch Surg 1969, 99: 612-614.

38. Antrum, R.M. Perianal haematoma: an unusual feature of a leaking aortic aneurysm. Br J Surg 1984, 71: 649.

39. Cullen, T.S. A new sign in ruptured extrauterine pregnancy. Am J Obstet 1918, 78: 457.

40. Armour, R.H., Clifton, M.A. \& Marsh, C.H. Balloon catheter control of a ruptured abdominal aortic aneurysm in a patient with Cullen's sign. Br J Surg 1978, 65: 350.

41. Grabowski, E.W. \& Pilcher, D.B. Ruptured abdominal aortic aneurysm manifesting as symptomatic inguinal hernia. Am Surg 1981, 47: 311-312.

42. Louras, J.C. \& Welch, J.P. Masking of ruptured abdominal aortic aneurysm by incarcerated inguinal hernia. Arch Surg 1984, 119: 331-332.

43. Khaw, H., Sottiuria, V.S., Craighead, C.G. \& Batson, R.C. Ruptured abdominal aortic aneurysm presenting as symptomatic inguinal mass: report of six cases. Vasc Surg 1986, 4: 384-386.

44. Banerjee, A.K., Bhutiani, R.P. \& Lewis, J.D. Irreducible inguinal hernia as a presentation of ruptured abdominal aortic aneurysm. J R Coll Surg Edin 1989, 34: 281.

45. Evans, D.M. \& Webster, J.H.H. Spontaneous aortoduodenal fistula. Br J Surg 1972, 59: 368-372.

46. Sweeney, M.S. \& Gadacz, T.R. Primary aortoduodenal fistual: Manifestation, diagnosis and treatment. Surgery 1984, 98: 492-497.

47. Perdue, G.D., Smith, R.B., Ansley, J.D. \& Costantino, M.J. Impending aortoenteric hemorrhage-the effect of early recognition on improved outcome. Ann Surg 1976, 192: 237-243.

48. Kleinman, L.H., Towne, J.B. \& Bernard, V.M. A diagnostic and therapeutic approach to aorto-enteric fistulas: Clinical experience with 20 patients. Surgery 1979, 86: 868-877.

49. Connolly, J.E., Kwaan, J.H., McCort, P.M., Brownell, D.A. \& Levine, E.F. Aortoenteric fistula. Ann Surg 1982, 194: 402-412.

50. Mehta, A.I., McDowell, D.E. \& James, E.C. Treatment of massive gastrointestinal hemorrhage from aortoenteric fistula. Surg Gynec Obstet 1978, 142: 59-62.

51. Reckless, J.P.D., McColl, I. \& Taylor, G.W. Aortoenteric fistula: An uncommon complication of abdominal aortic aneurysm. Br J Surg 1972, 59: 461-462.

52. Wilson, S.E. \& Owens, M.L. Aortocolic fistula, a lethal cause of lower gastrointestinal bleeding. Report of a case. Dis Colon Rectum 1976, 19: 614-617.

53. Reddy, K.R. \& Stillman, R.M. Spontaneous aortosigmoid fistula. JAMA 1982, 247: 2565-2566.

54. Hashmonai, M.H., Stahl, S. \& Schramek, A. Rupture of an abdominal aortic aneurysm preesnting as painful obstructive jaundice. J Cardiovasc Surg 1981, 22: 187-189.

55. Chandler, J.J. The Einstein sign: the clinical picture of acute cholecystitis caused by ruptured abdominal aortic aneurysm. $N$ Engl J Med 1984, 310: 1538. 
56. Alexander, J.J. \& Imbembo, A.L. Aorto-vena caval fistula. Surgery 1989, 105: 1-12.

57. Nennhaus, H.P. \& Javid, H. The distinct syndrome of spontaneous abdominal aortocaval fistula. Am J Med 1968, 44: 464-472.

58. Burke, A.M. \& Jamieson, G.G. Aortocaval fistula associated with ruptured aortic aneurysm. Br J Surg 1983, 70: 431-433.

59. Gordon, J.B., Newman, K.D. \& Marsh, J.D. Angina pectoris as the initial manifestation of an aortocaval fistula. $\mathrm{Am} \mathrm{J} \mathrm{Med}$ 1986, 80: 514-516.

60. Gertner, M.H., Hargrove, W.C. \& Roberts, H.B. A ruptured abdominal aortic aneurysm presenting as inferior vena caval obstruction. Surgery 1978, 83: 605-607.

61. Lord, J.W., Vigorita, J. \& Florio, J. Fistula between abdominal aortic aneurysm and anomalous renal vein. JAMA 1964, 187: 535-536.

62. Horan, D.P. \& Sharp, J.H. Spontaneous fistula between the aorta and left renal vein. Am J Surg 1967, 113: 802-806.
63. Yashar, J.J., Hallman, G.L. \& Cooley, D.A. Fistula between aneurysm of aorta and left renal vein. Arch Surg 1969, 99: $546-548$.

64. Celoria, G.M., Friedmann, P., Rhee, S.W. \& Berman, J. Fistulas between the aorta and the left renal vein. J Vasc Surg 1987, 6: 191-193.

65. Suzuki, M., Collins, G.M., Bassinger, G.T. \& Dilley, R. Aorto-left renal vein fistula: an unusual complication of abdominal aortic aneurysm. Ann Surg 1976, 184: 31-34.

66. Harjula, A., Luosto, R., Konttinen, M., Kiusaari, A. \& Mankinen, P. Fistulas between the aorta and the left renal vein. J Vasc Surg 1987, 6: 191-193.

67. Linsell, J.C., Rowe, P.H. \& Owen, W.J. Rupture of an aortic aneurysm into the renal vein presenting as a left-sided varicocele. Acta Chir Scand 1987, 153: 477-478.

(

